Spin Physics (SPIN2014)

International Journal of Modern Physics: Conference Series

Vol. 40 (2016) 1660034 (6 pages)

(C) The Author(s)

DOI: $10.1142 / \mathrm{S} 201019451660034 \mathrm{X}$

\title{
Extraction of Transversity from COMPASS and Belle Data
}

\author{
Franco Bradamante \\ In collaboration with Vincenzo Barone and Anna Martin \\ Dipartimento di Fisica, Trieste University, and INFN, Sezione di Trieste, \\ via A. Valerio 2, I-34127 Trieste, Italy \\ Franco.Bradamante@ts.infn.it \\ Published 29 February 2016
}

\begin{abstract}
The valence transversity distributions of the $\mathrm{u}$ - and the d-quarks have been extracted point-by-point from single-hadron production and dihadron production data measured in semi-inclusive deep inelastic scattering and in $e^{+} e$ annihilation. The transversity distributions are found to be compatible with each other and with previous analyses.
\end{abstract}

Keywords: Nucleon spin structure; transversity; COMPASS; BELLE.

PACS numbers: 13.60.-r, 13.88.+e, 14.20.-c, 14.65.-q

\section{Introduction}

The transversity distribution function $h_{1}$ was introduced already thirty five years ago $^{1}$ but it has been convincingly shown to be different from zero only recently, in the last ten years, when several measurements of semi-inclusive deep inelastic scattering (SIDIS) on transversely polarized targets have been performed at COMPASS, on deuterons and protons, at HERMES, on protons, and very recently in Hall A at JLab, on neutrons (for reviews see Refs. 2, 3). In those measurements target transverse spin dependent azimuthal asymmetries were observed in the hadrons produced in the current jet, which depend both on the transversity distribution of the struck quark and on a fragmentation function (FF). Two situations were hypothesized. In the first only one hadron in the current jet is observed, and the asymmetry is given by a convolution of the transversity function $h_{1}$ and a fragmentation function $H_{1}$, the so-called Collins FF, ${ }^{4}$ which depends on the transverse momentum of the fragmenting hadron. In the second, a pair of oppositely charged hadrons is observed, and the asymmetry is given by the product of the transversity distribution and another $\mathrm{FF}$, the dihadron $\mathrm{FF} H_{q}^{\angle .5,6}$ Both asymmetries have been

This is an Open Access article published by World Scientific Publishing Company. It is distributed under the terms of the Creative Commons Attribution 3.0 (CC-BY) License. Further distribution of this work is permitted, provided the original work is properly cited. 
measured to be different from zero in SIDIS experiments. Essential complementary information on the Collins and the dihadron FFs has been provided by the Belle Collaboration, which measured the correlations between the azimuthal angles of the hadrons belonging to two back to back jets in $e^{+} e^{-} \rightarrow q \bar{q} \rightarrow j \bar{j}$ which depends on the square of the relevant FF. Suitably parametrizing the parton distribution functions (PDFs) and the FFs global fits with SIDIS and $e^{+} e^{-}$data could separately extract the transversity distributions and the two FFs. ${ }^{2,7,8}$ In this work we adopt a different approach and extract the transversity PDF directly from the 1-h and 2-h SIDIS and $e^{+} e^{-}$data, without any parametrization of transversity PDFs and FFs, and consequently without performing any global fit. The strategy is to extract from the $e^{+} e^{-}$data the analyzing power of 1-h and 2-h production from a transversely polarized quark, and then use this information to obtain point-by-point the transversity distributions from the SIDIS data. The need to dispose of both proton and deuteron data measured at the same $x$ and $Q^{2}$ values limited the data set to the COMPASS data. Preliminary results on this work have already been presented ${ }^{9}$ and an extensive paper with the final analysis and results has been sent for publication soon after this Symposium, ${ }^{10}$ The present report only sketches rapidly the procedure, discussing first the 2 -h case and moving to the 1 -h case afterwards.

\section{Dihadron Asymmetries}

In SIDIS, after correcting for the spin transfer parameter, the differential transverse spin asymmetry for dihadron production is given by ${ }^{11,12}$

$$
A^{2 h}=\frac{\sum_{q, \bar{q}} e_{q}^{2} x h_{1}^{q} H_{q}}{\sum_{q, \bar{q}} e_{q}^{2} x f_{1}^{q} D_{q}} .
$$

where $f_{1}^{q}$ are the unpolarised PDFs, $D_{q}$ are the FFs for hadron pair production, and we have defined

$$
H_{q}\left(z, M_{2 h}\right)=\sin \theta \cdot R / M_{2 h} \cdot H_{q}^{<}\left(z, M_{2 h}\right) .
$$

with $M_{2 h}$ the invariant mass of the hadron pair, $z=z_{1}+z_{2}$ the fraction of longitudinal momentum carried by the two hadrons, $\theta$ the angle in the two hadron center of mass system, $\vec{R}=\left(\vec{P}_{1}-\vec{P}_{2}\right) / 2$, where $\vec{P}_{1,2}$ are the momenta of the two hadrons. With very reasonable assumptions ${ }^{11,12}$ and neglecting the $s$ and $c$ quark contribution, the asymmetries measured in COMPASS ${ }^{13,14}$ in the different $x$ bins can then be written as:

$$
\begin{aligned}
& A_{p}^{2 h} \simeq \frac{4 x h_{1}^{u_{v}}-x h_{1}^{d_{v}}}{4 x\left(f_{1}^{u}+f_{1}^{\bar{u}}\right)+x\left(f_{1}^{d}+f_{1}^{\bar{d}}\right)} \frac{<H_{u}>}{<D_{u}>}, \\
& A_{d}^{2 h} \simeq \frac{3}{5} \frac{x h_{1}^{u_{v}}+x h_{1}^{d_{v}}}{x\left(f_{1}^{u}+f_{1}^{\bar{u}}\right)+x\left(f_{1}^{d}+f_{1}^{\bar{d}}\right)} \frac{<H_{u}>}{<D_{u}>},
\end{aligned}
$$

where $\left\langle H_{u}\right\rangle$ and $\left\langle D_{u}\right\rangle$ are the FFs of the $u$ quark integrated over $z$ and $M_{2 h}$. The values of the COMPASS asymmetries we have used in this analysis are taken 
from Refs. 13, 14 and we have assumed that the charged hadrons are pions, which is a good approximation. The combinations of the unpolarised PDFs have been computed using CTEQ5D thus the only missing piece to extract the transversity combinations from eq. (3) is the analysing power $\left\langle a_{P}\right\rangle=\left\langle H_{u}\right\rangle /\left\langle D_{u}\right\rangle$.

The extraction of the analysing power from the Belle data is very similar to what has been done by the Pavia group in their early paper on this subject. ${ }^{11}$

In $\mathrm{e}^{+} \mathrm{e}^{-} \rightarrow q \bar{q} \rightarrow j \bar{j}$ the asymmetry can be written as ${ }^{12,15}$

$$
a_{12}=\frac{s^{2}}{1+c^{2}} \frac{\sum_{q} e_{q}^{2} H_{q} H_{\bar{q}}}{\sum_{q} e_{q}^{2} D_{q} D_{\bar{q}}} \simeq \frac{s^{2}}{1+c^{2}} \frac{5}{8} \frac{H_{u}^{2}}{D_{u}^{2}}
$$

where the "bar" quantities refer to the jet initiated by the $\bar{q}$, and $s^{2}=\sin ^{2} \theta_{2}$ and $c^{2}=\cos ^{2} \theta_{2}$, with $\theta_{2}$ the angle between the $e^{+}$and the pair direction. We have used the previous assumptions on the FFs and $D_{c}=0.5 D_{u}$. From the fully integrated asymmetry measured by Belle, ${ }^{15} a_{12}^{I}=-0.0196 \pm 0.0002 \pm 0.0022$, the analysing power turns out to be $\left\langle a_{P}\right\rangle=-0.203$. The value has been taken as negative in order to get a positive transversity for the $u$ quark.

From eq. (3) it is now possible to compute the quantities $4 h_{1}^{u_{v}}-h_{1}^{d_{v}}$ and $h_{1}^{u_{v}}+h_{1}^{d_{v}}$ which are shown in Fig. 1 and, from these, the transversity PDFs $x h_{1}^{u_{v}}$ and $x h_{1}^{d_{v}}$ whose preliminary values are shown as closed squares in Fig. 3 together with the corresponding quantities extracted from the Collins asymmetries as described in the next section.

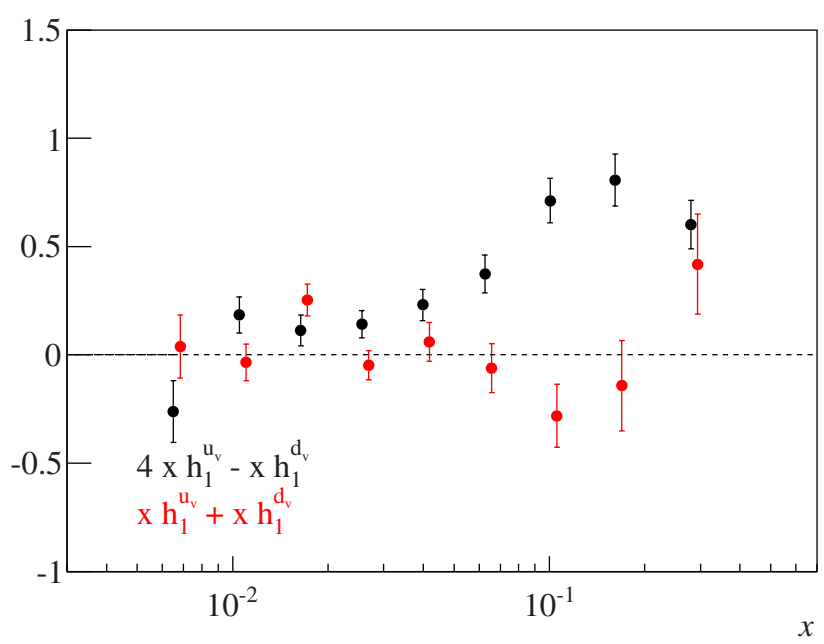

Fig. 1. Preliminary values of $4 x h_{1}^{u_{v}}-x h_{1}^{d_{v}}$ (from p data) and $x h_{1}^{u_{v}}+x h_{1}^{d_{v}}$ (from d data). 


\section{Collins Asymmetries}

The notation we use is the same as in ref.. ${ }^{16,17}$ For convenience we introduce

$$
H_{1 q}^{ \pm}=H_{1\left(q \rightarrow \pi^{ \pm}\right)}^{\perp(1 / 2)}, \quad D_{1 q}^{ \pm}=D_{1\left(q \rightarrow \pi^{ \pm}\right)},
$$

and we consider only $\pi^{+}$and $\pi^{-}$in the final state. We use in the following the experimental SIDIS asymmetries for charged hadrons from the entire data set of COMPASS with the deuteron ${ }^{18}$ and with the proton target. ${ }^{19}$

To calculate the analysing power from the Belle data we have used the so-called $A_{12}^{U L}$ asymmetry ${ }^{20}$ measured for $z_{1}=z_{2}=z$ in $4 z$ bins. With the usual assumptions on the FFs, the asymmetry can be written as

$$
A_{12}^{U L}(z)=\frac{<s^{2}>}{<1+c^{2}>}\left[\frac{H_{1}^{f a v}(z)}{D_{1}^{f a v}(z)}\right]^{2} B(z)
$$

where the favored and disfavored FFs have the usual definition, namely

$$
\begin{aligned}
& H_{1}^{f a v}=H_{1 u}^{+}=H_{1 d}^{-}=H_{1 \bar{u}}^{-}=H_{1 \bar{d}}^{+} \\
& H_{1}^{d i s}=H_{1 u}^{-}=H_{1 d}^{+}=H_{1 \bar{u}}^{+}=H_{1 \bar{d}}^{-}
\end{aligned}
$$

and correspondingly for the $D_{1 q}^{ \pm}$FFs. $B(z)$ depends on the ratios

$$
a(z)=\frac{H_{1}^{\text {dis }}(z)}{H_{1}^{\text {fav }}(z)}, \quad b(z)=\frac{D_{1}^{\text {dis }}(z)}{D_{1}^{\text {fav }}(z)} .
$$

Only the $b(z)$ values can be obtained by the standard FF parametrisations, while $a(z)$ is not known. To evaluate the analysing power $A_{P, B}(z)=H_{1}^{f a v}(z) / D_{1}^{f a v}(z)$ in the $4 z$ bins we have done two alternative assumptions:

$$
\begin{aligned}
& A: \quad a(z)=-1, \quad \text { i.e. } \quad H_{1}^{\text {dis }}(z)=-H_{1}^{\text {fav }}(z) \\
& B: \quad a(z)=-b(z), \text { i.e. } H_{1}^{\text {dis }}(z) / D_{1}^{\text {dis }}(z)=-H_{1}^{f a v}(z) / D_{1}^{\text {fav }}(z) .
\end{aligned}
$$

The measured values of the analyzing power and the fitting functions are shown in Fig. 2. After integration over $z$ one gets for the two hypothesis

$$
A:<H_{1}^{f a v}>/<D_{1}^{f a v}>\simeq 0.12, \quad B:<H_{1}^{f a v}>/<D_{1}^{f a v}>\simeq 0.17 .
$$

The Collins asymmetry for $\pi^{ \pm}$in SIDIS can be written as

$$
A_{\text {Coll }}^{ \pm}(x, z)=\frac{\sum_{q, \bar{q}} e_{q}^{2} x h_{1}^{q}(x) \otimes H_{1 q}^{ \pm}(z)}{\sum_{q, \bar{q}} e_{q}^{2} x f_{1}^{q}(x) \otimes D_{1 q}^{ \pm}(z)}=C_{G} \cdot \frac{\sum_{q, \bar{q}} e_{q}^{2} x h_{1}^{q}(x) H_{1 q}^{ \pm}(z)}{\sum_{q, \bar{q}} e_{q}^{2} x f_{1}^{q}(x) D_{1 q}^{ \pm}(z)}
$$

using the "Gaussian ansatz". ${ }^{16}$ In the following we assume $C_{G}=1$, a reasonable assumption at low $z$, where the statistics is highest. 

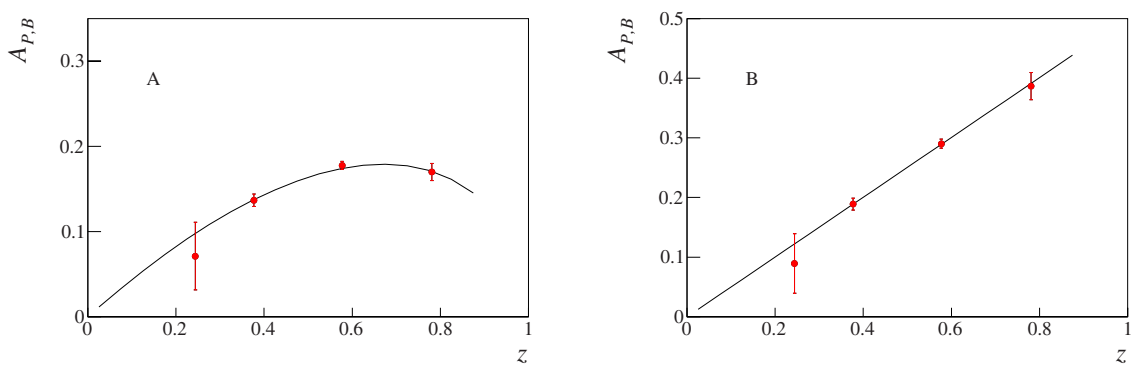

Fig. 2. The analysing power $A_{P, B}(z)=H_{1}^{f a v}(z) / D_{1}^{f a v}(z)$ as function of $z$ for $a=-1$ (right) and $a=-b$ (left).

When only the $x$ dependence of $A_{C o l l}^{ \pm}$is considered, and neglecting the $s$ and $c$ quark contributions, one gets

$$
A_{\text {Coll }, p}^{+}=\frac{<H_{1}^{f a v}>}{<D_{1}^{\text {fav }}>} \frac{4\left(x h_{1}^{u}+\alpha x h_{1}^{\bar{u}}\right)+\left(\alpha x h_{1}^{d}+x h_{1}^{\bar{d}}\right)}{d_{p}^{+}}
$$

where $\alpha=<H_{1}^{\text {dis }}>/<H_{1}^{f a v}>$, and the quantity $d_{p}^{+}$corresponds to the numerator when evaluated with unpolarised PDFs and FFs. The analysing power $\left\langle H_{1}^{f a v}\right\rangle$ $/<D_{1}^{\text {fav }}>$ values are those obtained from Belle data and given in (9). Similar expressions, containing different combinations of the PDFs have been written for $A_{\text {Coll }, p}^{-}, A_{C o l l, d}^{+}$and $A_{\text {Coll }, d}^{-}$.

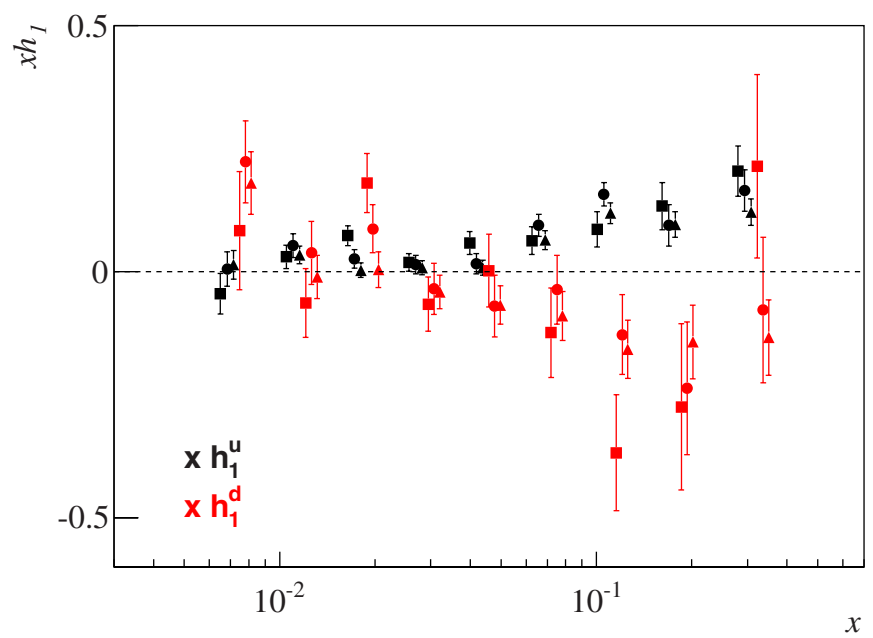

Fig. 3. Preliminary results for the transversity PDFs for u-quark (black points) and d-quark (red points) from dihadron (squares) and Collins asymmetries (circles for I1, triangles for I2). 
In this preliminary work $\bar{q}$ transversity was neglected (on the contrary, in Ref. ${ }^{10}$ it was extracted from the data). Solving the four equations, in the two different assumptions for $a(z)$ and $\alpha$, we got the preliminary values for the valence quarks transversity PDFs given in Fig. 3. The values in the figure have been obtained neglecting $Q^{2}$ evolution of the spin dependent FFs which at least for the 2 -h case is known to be small.

\section{Conclusions}

In spite of the large difference in the analysing power in the two hypothesis $\mathrm{A}$ and $\mathrm{B}$, the extracted transversity values from the Collins asymmetries are remarkably close and in very good agreement with the corresponding quantities evaluated from the dihadron asymmetries. The u-quark transversity is definitively different from zero and is determined rather well, thanks to the larger amount of data collected by the COMPASS Collaboration with the proton target as compared to the deuteron target. The d-quark transversity has larger statistical errors, but in our extraction it is also definitively different from zero, and has roughly the same size and opposite sign as compared to the u-quark transversity. As a further remark, it can be safely stated that the $Q^{2}$ evolution affects in a similar way the Collins and the dihadron asymmetries. Also, the effect of the convolution over the transverse momenta, which is present in the Collins asymmetry, seems to be small.

\section{References}

1. J. P. Ralston and D. E. Soper, Nucl. Phys. B152, 109 (1979).

2. V. Barone, F. Bradamante and A. Martin, Prog. Part. Nucl. Phys. 65267 (2010).

3. C. A. Aidala, S. D. Bass, D. Hasch and G. K. Mallot, Rev. Mod. Phys. 85, 655 (2013).

4. J.C. Collins, Nucl. Phys. B396, 161 (1993).

5. J.C. Collins and G.A. Ladinsky, hep-ph/9411444.

6. R. L. Jaffe, X.-m. Jin, and J. Tang, Phys. Rev. Lett. 80, 1166 (1998).

7. M. Anselmino et al., Phys. Rev. D 87094019 (2013) and references therein.

8. A. Bacchetta, A. Courtoy and M. Radici, JHEP 1303119 (2013) and references therein.

9. F. Bradamante, proceedings of QCD Evolution Workshop, May 12-16, 2014 Santa Fe, NM.

10. A. Martin, F. Bradamante and V. Barone, Phys. Rev. D 91014034 (2015).

11. A. Bacchetta, A. Courtoy and M. Radici, Phys. Rev. Lett. 107012001 (2011).

12. A. Courtoy, A. Bacchetta, M. Radici and A. Bianconi, Phys. Rev. D 85114023 (2012).

13. C. Adolph et al. [COMPASS Collaboration], Phys. Lett. B 71310 (2012).

14. C. Adolph et al. [COMPASS Collaboration], Phys. Lett. B 736124 (2014).

15. A. Vossen et al. [Belle Collaboration], Phys. Rev. Lett. 107072004 (2011).

16. A. V. Efremov, K. Goeke and P. Schweitzer, Phys. Rev. D 73094025 (2006).

17. A. Bacchetta, L. P. Gamberg, G. R. Goldstein and A. Mukherjee, Phys. Lett. B 659 234 (2008).

18. E. S. Ageev et al. [COMPASS Collaboration], Nucl. Phys. B 76531 (2007).

19. C. Adolph et al. [COMPASS Collaboration], Phys. Lett. B 717376 (2012).

20. R. Seidl et al. [Belle Collaboration], Phys. Rev. D 78032011 (2008) [Erratum-ibid. D 86039905 (2012)]. 